\title{
Minigap, Parity Effect and Persistent Currents in SNS Nanorings
}

\author{
Mikhail S. Kalenkov ${ }^{1,2}$, Harald Kloos ${ }^{1}$ and Andrei D. Zaikin ${ }^{1,2}$ \\ ${ }^{1}$ Forschungszentrum Karlsruhe, Institut für Nanotechnologie, 76021, Karlsruhe, Germany \\ ${ }^{2}$ I.E. Tamm Department of Theoretical Physics, P.N. Lebedev Physics Institute, 119991 Moscow, Russia
}

\begin{abstract}
We have evaluated a proximity-induced minigap in the density of states (DOS) of SNS junctions and SNS nanorings at an arbitrary concentration of non-magnetic impurities. We have demonstrated that an isotropic energy minigap in the electron spectrum opens up already at arbitrarily weak disorder, while angle resolved DOS at higher energies can remain strongly anisotropic. The minigap value $\varepsilon_{g}$ can be tuned by passing a supercurrent through an SNS junction or by applying a magnetic flux $\Phi$ to an SNS ring. A non-monotonous dependence of $\varepsilon_{g}$ on $\Phi$ has been found at weak disorder. We have also studied persistent currents in isolated SNS nanorings. For odd number of electrons in the ring we have found a non-trivial current-phase (current-flux) relation which - at relatively high disorder - may lead to a $\pi$-junction state and spontaneous currents in the ground state of the system.
\end{abstract}

\section{INTRODUCTION}

In hybrid structures composed of a superconductor $(\mathrm{S})$ and a normal metal $(\mathrm{N})$ Cooper pairs can penetrate into the latter thereby significantly changing the properties of the system ${ }^{1}$. As a result of this proximity effect the $\mathrm{N}$-metal also acquires superconducting properties being able to carry supercurrent and, hence, exhibiting Josephson ${ }^{2.3 .4}$ and Meissner ${ }^{5.6}$ effects.

Another interesting consequence of this proximityinduced superconductivity is the existence of a minigap in the electron spectrum of a normal metal. In the diffusive limit this minigap $\varepsilon_{g}$ was found to be of order of the Thouless energy $\varepsilon_{\mathrm{Th}}$ of this metal ${ }^{7,8,9,10}$. In strictly ballistic SN and SNS systems this gap is strongly anisotropic ${ }^{11}$, and it vanishes for electrons propagating parallel to the SN interface. For arbitrary concentration of non-magnetic impurities the minigap dependence on the electron elastic mean free path was studied in Ref. 12 . In SNS structures the proximity-induced minigap $\varepsilon_{g}$ can be tuned by applying the phase difference $\varphi$ across the N-metal ${ }^{2,13}$.

Modifications of the normal metal density of states (DOS) due to the proximity effect can be - and were studied experimentally with the aid of tunneling spectroscopy methods, see, e.g., Ref. 14. In this paper we point out that the proximity-induced minigap in SN sandwiches can be directly measured with the aid of the superconducting parity effect ${ }^{15.16 .17}$. Indeed, the ground state energies of isolated superconducting system with odd and even electron numbers should differ exactly by the value of the gap in the electron spectrum. In the case of superconducting grains it is just the BCS gap, while in SN and SNS structures this value should be set by the proximity-induced minigap $\varepsilon_{g}$. Hence, the latter can be directly observed in an experimental setup similar to that used, e.g., in Ref. 18 .

The possibility of tuning the minigap value $\varepsilon_{g}(\varphi)$ by passing the supercurrent through SNS junctions provides additional ways to independently test theoretical predictions. One can, for instance, consider an isolated su-

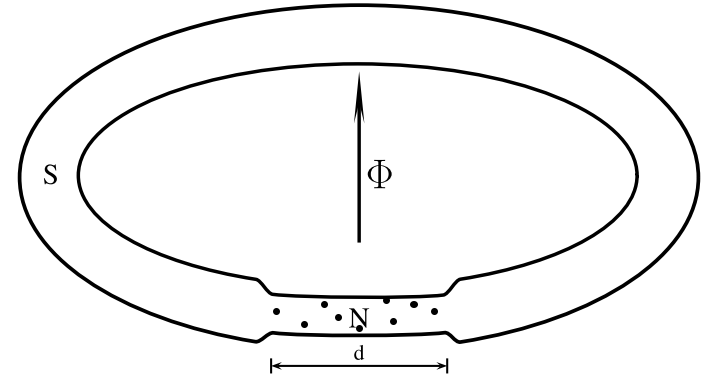

FIG. 1: A superconducting ring with embedded normal metal of length $d$.

perconducting ring with an embedded layer of a normal metal, the so-called SNS ring ${ }^{19}$. This system is depicted in Fig. 1. Applying an external magnetic flux $\Phi$ to such a system one induces persistent currents (PC) circulating inside the ring. Both the magnitude and the flux dependence of such currents will depend on the parity of the total number of electrons in the ring ${ }^{19,20}$. The difference between PC values for odd $\left(I_{o}\right)$ and even $\left(I_{e}\right)$ ensembles is related to the minigap value $\varepsilon_{g}(\varphi)$. This relation acquires a particularly simple form in the limit $T \rightarrow 0$ in which case one finds 19

$$
I_{o}(\Phi)=I_{e}(\Phi)+2 e \frac{\partial \varepsilon_{g}(\varphi)}{\partial \varphi}
$$

The last term in this equation describes the contribution to the current from the "odd" electron occupying the lowest available state above the minigap $\varepsilon_{g}(\varphi)$ in the density of states of the normal metal.

For a broad range of system parameters the phase difference $\varphi$ across the SNS junction is linked to the external magnetic flux $\Phi$ by means of the standard relation $\varphi=2 \pi \Phi / \Phi_{0}\left(\Phi_{0}\right.$ is the flux quantum) which will also be assumed to hold throughout this paper. Eq. (1) can be used, on one hand, for an independent study of the phase dependence of the minigap $\varepsilon_{g}(\varphi)$ and, on the other hand, for further investigations of the parity-affected persistent currents in SNS nanorings. 
The structure of our paper is as follows. In Sec. II we will employ the quasiclassical formalism of Eilenberger equations and evaluate the phase-dependent minigap $\varepsilon_{g}(\varphi)$ in SNS systems at an arbitrary concentration of non-magnetic impurities. We will demonstrate that an isotropic minigap opens up in the normal metal already in the limit of very weak disorder. At sufficiently large values of the electron elastic mean free paths we recover a non-monotonous dependence of the minigap on $\varphi$. In Sec. III we will analyze an interplay between parity effect and persistent currents in SNS nanorings with an arbitrary impurity concentration. We will show that in the case of odd total number of electrons in the ring the dependence of $\mathrm{PC}$ on the applied magnetic flux acquires non-trivial features which might lead to a $\pi$-junction behavior and spontaneous currents in the ground state of the ring. A brief discussion of our key observations is presented in Sec. IV.

\section{PHASE-DEPENDENT MINIGAP AT ARBITRARY IMPURITY CONCENTRATIONS}

Let us analyze thermodynamic properties of SNS rings presented in Fig. 10 We will stick to the simplest case of a superconductor with singlet isotropic pairing and assume that both NS interfaces are fully transparent. The length of the normal metal layer and its cross section are denoted respectively as $d$ and $\mathcal{A}$.

Our analysis is based on the Eilenberger equations ${ }^{10.21}$ for the energy-integrated retarded $2 \times 2$ matrix Green functions $\hat{g}$

$$
\begin{gathered}
{\left[\varepsilon \hat{\tau}_{3}-\hat{\Delta}(\boldsymbol{r})-\hat{\Sigma}(\boldsymbol{r}, \varepsilon), \hat{g}\left(\boldsymbol{p}_{F}, \boldsymbol{r}, \varepsilon\right)\right]=-i \boldsymbol{v}_{F} \nabla \hat{g}\left(\boldsymbol{p}_{F}, \boldsymbol{r}, \varepsilon\right),} \\
\hat{g}^{2}\left(\boldsymbol{p}_{F}, \boldsymbol{r}, \varepsilon\right)=1,
\end{gathered}
$$

where $[\hat{a}, \hat{b}]=\hat{a} \hat{b}-\hat{b} \hat{a}, \varepsilon$ is the quasiparticle energy, $\boldsymbol{p}_{F}=$ $m \boldsymbol{v}_{F}$ is the electron Fermi momentum vector and $\hat{\tau}_{3}$ is the Pauli matrix. The matrices $\hat{g}$ and $\hat{\Delta}$ have the standard form

$$
\hat{g}=\left(\begin{array}{cc}
g & f \\
f^{+} & -g
\end{array}\right), \quad \hat{\Delta}=\left(\begin{array}{cc}
0 & \Delta \\
-\Delta^{*} & 0
\end{array}\right)
$$

where $\Delta$ is the BCS superconducting order parameter chosen to be spatially constant in the superconductor and equal to zero in the normal metal. Electron scattering on non-magnetic impurities is accounted for by the selfenergy term $\hat{\Sigma}$. Within the Born approximation one has

$$
\hat{\Sigma}(\boldsymbol{r}, \varepsilon)=-i \frac{v_{F}}{2 \ell}\left\langle\hat{g}\left(\boldsymbol{p}_{F}, \boldsymbol{r}, \varepsilon\right)\right\rangle,
$$

where $\ell$ is the electron elastic mean free path and angular brackets $\langle\ldots\rangle$ denote averaging over the Fermi momentum directions.

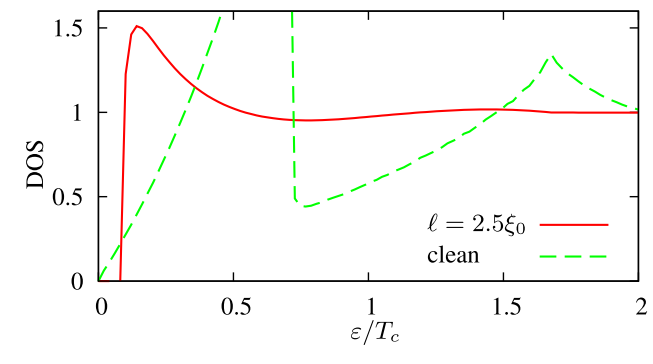

FIG. 2: Angle averaged DOS in the middle of the normal layer. Here and below we choose $d=10 \xi_{0}$.

The current density and the angle resolved local density of states are defined as

$$
\begin{gathered}
\boldsymbol{j}(\boldsymbol{r})=e N_{0} \operatorname{Re} \int_{-\infty}^{\infty} d \varepsilon \tanh \left(\frac{\varepsilon}{2 T}\right)\left\langle\boldsymbol{v}_{F} g\left(\boldsymbol{p}_{F}, \boldsymbol{r}, \varepsilon\right)\right\rangle, \\
\nu\left(\boldsymbol{p}_{F}, \boldsymbol{r}, \varepsilon\right)=\operatorname{Re} g\left(\boldsymbol{p}_{F}, \boldsymbol{r}, \varepsilon\right),
\end{gathered}
$$

where $\operatorname{DOS} \nu\left(\boldsymbol{p}_{F}, \boldsymbol{r}, \varepsilon\right)$ is normalized to its normal state value at the Fermi energy $N_{0}=m p_{F} / 2 \pi^{2}$. Let us also note that the above quasiclassical formalism and, in particular, the description of electron scattering in terms of the self-energy (5.) are applicable provided the number of conducting channels in the system is large $N_{C h}=\mathcal{A} p_{F}^{2} / 4 \pi \gg 1$. This inequality will be assumed to hold throughout the whole calculation ${ }^{22}$. In addition, we will assume that the length of the N-metal strongly exceeds the superconducting coherence length $d \gg \xi_{0} \sim v_{F} / \Delta$.

Our first goal is to analyze the behavior of DOS $\nu\left(\boldsymbol{p}_{F}, \boldsymbol{r}, \varepsilon\right)$ in the normal metal as a function of the phase difference $\varphi=2 \pi \Phi / \Phi_{0}$ for arbitrary values of elastic mean free path $\ell$. To begin with, let us recall that in a strictly ballistic limit $\ell \rightarrow \infty$ the spectrum of an SNS junction below the BCS energy gap is formed by discrete Andreev levels with energies $E_{n}$. In the limit $E_{n} \ll \Delta$ the values $E_{n}$ read 2.11

$$
E_{n}=\frac{\left|v_{x}\right|}{2 d}\left[\pi(2 n+1)+\varphi \operatorname{sgn} v_{x}\right],
$$

where $v_{x}$ is the $x$-component of the Fermi velocity vector $\boldsymbol{v}_{F}$. This expression demonstrates that the proximity induced gap in the spectrum of a ballistic SNS system is strongly anisotropic. It depends on the direction of the Fermi velocity and vanishes for electrons propagating parallel to NS interfaces. In other words, the trajectories for such electrons do not cross NS interfaces and, hence, these electrons do not suffer Andreev reflection and do not "feel" any proximity effect.

The situation changes significantly in the presence of already very weak disorder. In this case all electrons in the N-metal get scattered by non-magnetic impurities and, sooner or later, their trajectories hit one of the NS interfaces and electrons get Andreev-reflected. As a 


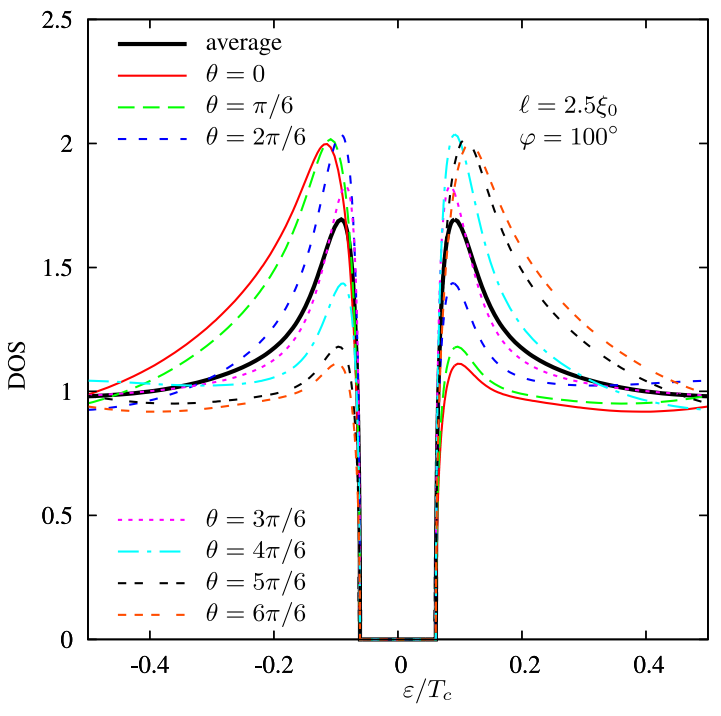

FIG. 3: The angle resolved DOS in the middle of the normal layer (thin lines) together with the angle averaged DOS (bold solid line). The angle between quasiparticle momentum and the $x$-direction (normal to NS interfaces) is denoted as $\theta$.

result, an isotropic proximity-induced minigap $\varepsilon_{g}(\varphi)$ develops in the density of states of the normal metal. The value of this minigap depends both on the electron mean free path $\ell$ and the phase difference $\varphi$ but it remains independent of the momentum direction for all values of $\ell$ and $\varphi$.

This property is illustrated in Figs. 2 and 3 Fig. 2 shows DOS inside the normal metal with disorder together with that evaluated in the ballistic limit $\ell \rightarrow \infty$. An important difference between the two curves is observed at low energies: The proximity-induced minigap is strictly zero in the ballistic limit while it opens up in the presence of disorder. At higher energies electron scattering on disorder broadens and eventually washes out peaks corresponding to Andreev levels (8).

Fig. 3 displays a typical energy dependence of the angle resolved density of states $\nu\left(\boldsymbol{p}_{F}, \boldsymbol{r}, \varepsilon\right)$ inside the normal layer of an SNS structure in the presence of disorder. We observe that $\nu\left(\boldsymbol{p}_{F}, \boldsymbol{r}, \varepsilon\right)$ vanishes for all $\varepsilon<\varepsilon_{g}(\varphi)$ independently of the direction of the Fermi momentum. Thus, already very weak disorder ${ }^{23}$ makes the minigap isotropic for all values of $\varphi$. In addition, we have verified that the minigap value does not depend on the coordinate inside the normal metal.

At the same time at energies just above the minigap $\varepsilon>\varepsilon_{g}$ the anisotropy in the density of states is clearly observable for sufficiently large values of the electron mean free path $\ell$, see Fig. 3 This anisotropy decreases with decreasing mean free path $\ell$, and in the dirty limit $\ell \lesssim \xi_{0}$ the density of states $\nu\left(\boldsymbol{p}_{f}, \boldsymbol{r}, \varepsilon\right)$ becomes almost isotropic. Anisotropic behavior of the density of states in the ultraclean limit $\ell \gg d$ can easily be understood if one observes that the minigap value in the latter limit is of order $\varepsilon_{g} \sim v_{F} / \ell \ll v_{F} / d$. Hence, for quasiparticles with

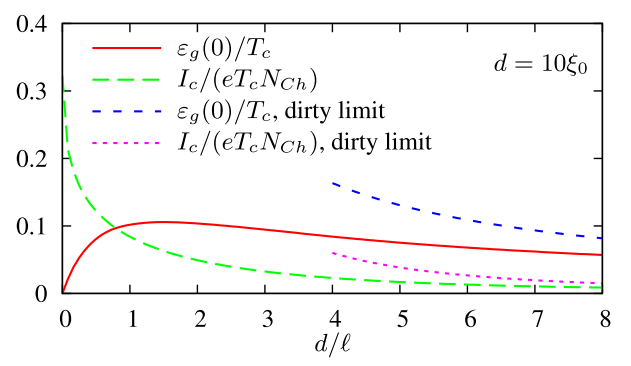

FIG. 4: The minigap $\varepsilon_{g}(0)$ and the zero temperature Josephson critical current of an SNS junction as a function of $d / \ell$. For sufficiently short values of $\ell$ both quantities approach the corresponding results derived in the diffusive limit from the Usadel equation analysis.

energies just above the minigap $v_{F} / d \gg \varepsilon \gtrsim v_{F} / \ell$ one can estimate $v_{x} \sim v_{F} d / \ell \ll v_{F}$, i.e. these states are formed predominantly by grazing electrons.

Let us also note that in the normal metal at energies just above the minigap $\varepsilon>\varepsilon_{g}$ the angle averaged DOS $\nu(\boldsymbol{r}, \varepsilon)=\left\langle\nu\left(\boldsymbol{p}_{F}, \boldsymbol{r}, \varepsilon\right)\right\rangle$ depends on energy as

$$
\nu(\boldsymbol{r}, \varepsilon) \propto\left(\varepsilon-\varepsilon_{g}\right)^{1 / 2} .
$$

This square-root dependence appears to be universal for all mean free path values $\ell$. In order to verify this statement we have plotted $\ln \nu(\varepsilon)$ versus logarithm of the parameter $\left(\varepsilon-\varepsilon_{g}\right) / \varepsilon_{g}$ for different values of $\ell$ (not shown). For all impurity concentrations at energies above the minigap our numerical data points collapse on straight lines corresponding to the same slope $1 / 2$. For very clean systems the dependence (9) is observed within narrower energy interval above the gap, and in the limit $\ell \rightarrow \infty$ this interval shrinks to zero faster than the minigap itself. On the contrary, for dirtier systems Eq. (9) applies for broader energy intervals and match with the corresponding dependence $\frac{13}{\underline{\underline{n}}}$ established from Usadel equations in the diffusive limit.

The dependence of the minigap $\varepsilon_{g}$ at $\varphi=0$ on the mean free path $\ell$ is depicted in Fig. 4. We have evaluated the minigap at $d=10 \xi_{0}$ and $d=10^{3} \xi_{0}$ and found a very good agreement with earlier numerical results of Pilgram et al ${ }^{12}$ obtained for NS structures with the normal layer thickness equal to $10^{2} \xi_{0}$. Our numerical results for $\varepsilon_{g} \equiv$ $\varepsilon_{g}(0)$ are rather well approximated by a simple analytical formula

$$
\varepsilon_{g}=\frac{v_{F}}{d} \frac{a y}{b y^{2}+c y+1},
$$

where $y=d / \ell$ and $a \approx 0.47, b \approx 0.45, c \approx 1.45$. With decreasing $\ell$ the minigap first grows as $\varepsilon_{g} \approx 0.47 v_{F} / \ell$, reaches its maximum $\varepsilon_{g}^{\max } \approx 0.17 v_{F} / d$ at $\ell \approx 0.67 d$ and then decays for small $\ell$ as $\varepsilon_{g} \approx 3.12 \varepsilon_{\mathrm{Th}}=1.04 v_{F} \ell / d^{2}$ approaching the results of Belzig et al $!^{8.10}$ derived from the Usadel equations analysis. Here and below $\varepsilon_{\mathrm{Th}}=$ $v_{F} \ell / 3 d^{2}$ is the Thouless energy of a normal metal.

Phase dependence of the minigap is shown in Fig. 5 for the mean free path values ranging between quasi-ballistic 


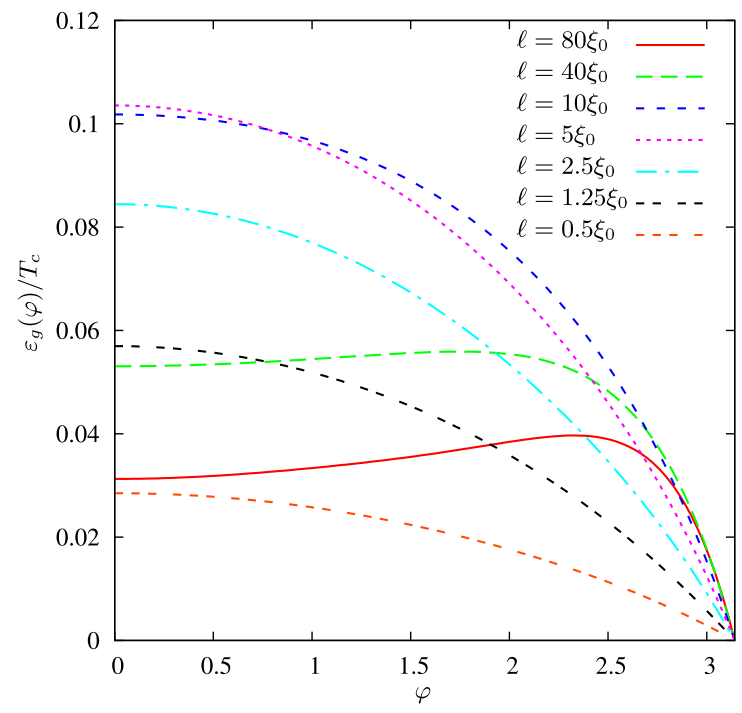

FIG. 5: Phase dependence of the minigap $\varepsilon_{g}(\varphi)$ for an SNS structure at different values of the electron mean free path $l$.

$(\ell \gg d)$ and diffusive $(\ell \ll d)$ regimes. A somewhat unexpected non-monotonous dependence of the minigap value on the phase difference is observed in the ultraclean limit $\ell \gg d$. We believe that this effect is caused by a non-trivial redistribution in the angle resolved DOS in the presence of the phase twist $\varphi$ across the N-layer. With decreasing the mean free path the maximum of the function $\varepsilon_{g}(\varphi)$ moves away from the point $\varphi=\pi$ towards smaller values of the phase difference. Eventually the maximum of $\varepsilon_{g}(\varphi)$ reaches the point $\varphi=0$ and this dependence becomes monotonous at all smaller values of $\ell$. In the diffusive limit $\ell \ll d$ our results approach those obtained by Zhou et al ${ }^{13}$ from Usadel equations, cf. Fig. 2 in Ref. 13 .

In the limit $\ell \ll d$ the minigap dependence on $\varphi$ is rather well approximated by a simple parabola

$$
\varepsilon_{g}(\varphi) \simeq \varepsilon_{g}(0)\left(1-\varphi^{2} / \pi^{2}\right) .
$$

Note that although this dependence is in agreement with our numerical results as well as with those displayed in Fig. 2 of Ref. 13, it does not agree with Eq. (5) from that paper at small phases $\varphi \ll \pi$.

\section{PERSISTENT CURRENTS AND PARITY EFFECT}

We now go over to the calculation of persistent currents in isolated SNS nanorings. As the total number of electrons in the ring is fixed to be either even or odd it is necessary to account for the parity effect. This task will be accomplished within the parity projection technique ${ }^{24.25 .26}$ recently adapted ${ }^{19.20}$ to the calculation of PC in superconducting nanorings.

Let define and evaluate the even/odd parity projected thermodynamic potentials

$$
\Omega_{e / o}=\Omega_{f}-T \ln \left[\frac{1 \pm e^{-A}}{2}\right], \quad A=\left(\Omega_{b}-\Omega_{f}\right) / T
$$

where $\Omega_{f}$ is the standard grand canonical thermodynamic potential and $\Omega_{b}$ is obtained from $\Omega_{f}$ by expressing the latter as a sum over the Fermi Matsubara frequencies $\omega_{f}=\pi T(2 n+1)$ with subsequent substitution of $\omega_{f}$ by the Bose frequencies $\omega_{b}=2 \pi T n$. For further details we refer the reader to the paper ${ }^{25}$.

With the aid of the Eilenberger quasiclassical formalism ${ }^{21}$ it is easy to cast the grand canonical thermodynamic potential of the system $\Omega_{f}$ to the following form

$$
\Omega_{f}=\tilde{\Omega}-4 N_{0} T \int d \boldsymbol{r} \int_{0}^{\infty} d \varepsilon \ln [2 \cosh (\varepsilon / 2 T)] \nu(\boldsymbol{r}, \varepsilon),
$$

where $\tilde{\Omega}=\int d \boldsymbol{r}\left|\Delta^{2}(\boldsymbol{r})\right| / \lambda+\sum_{\boldsymbol{k}} \xi_{\boldsymbol{k}}, \lambda$ is the BCS coupling constant and $\xi_{\boldsymbol{k}}$ is the single particle energy. Similarly, for $\Omega_{b}$ we find

$$
\Omega_{b}=\tilde{\Omega}-4 N_{0} T \int d \boldsymbol{r} \int_{0}^{\infty} d \varepsilon \ln [2 \sinh (\varepsilon / 2 T)] \nu(\boldsymbol{r}, \varepsilon) .
$$

With the aid of the above expressions it is now possible to directly evaluate PC circulating in a superconducting ring both for even and odd electron ensembles. Taking the derivative of $\Omega_{f / b}$ with respect to the phase difference one finds 19.20 :

$$
I_{e / o}=I_{f} \pm \frac{I_{b}-I_{f}}{\exp (A) \pm 1}
$$

where the upper (lower) sign corresponds to the even (odd) ensemble and

$$
I_{e / o}=2 e \frac{\partial \Omega_{e / o}}{\partial \varphi}, \quad I_{f / b}=2 e \frac{\partial \Omega_{f / b}}{\partial \varphi} .
$$

It is well known that the parity effect is mostly pronounced in the low temperature limit in which case the parameter $A$ (12) is much larger than one ${ }^{25} A \gg 1$. Evaluating the integrals in Eqs. (13), (14) at temperatures $T \ll\left|\varepsilon_{g}(\varphi)\right|$ we obtain

$$
A(T)=A_{N}(T)+A_{S}(T),
$$

where

$$
\begin{gathered}
A_{N}(T) \sim N_{0} V_{N} T^{3 / 2} \varepsilon_{g}^{-1 / 2}(\varphi) \exp \left(-\varepsilon_{g}(\varphi) / T\right), \\
A_{S}(T) \sim N_{0} V_{S} T^{1 / 2} \Delta^{1 / 2} \exp (-\Delta / T)
\end{gathered}
$$

are the contributions respectively from the normal and superconducting parts of the ring with the corresponding volumes $V_{N}$ and $V_{S}$. The term $A_{N}$ was evaluated making use of the dependence (9) for DOS at energies $\varepsilon$ just above the minigap. 


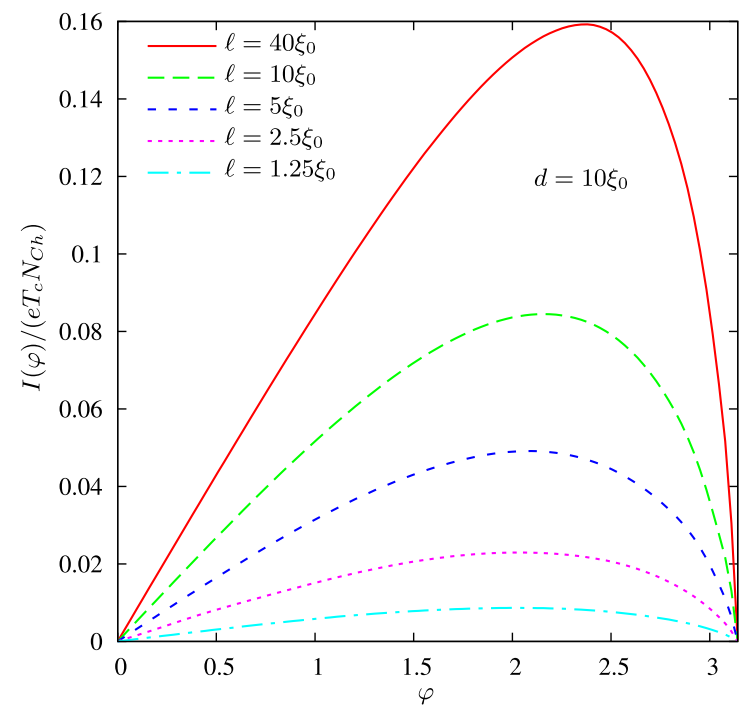

FIG. 6: Phase dependence for the zero temperature Josephson current $I(\varphi)$ at different values of the mean free path $l$.

Since throughout this paper we always assume the minigap to be smaller than the superconducting gap, $\varepsilon_{g}(\varphi)<\Delta$, at sufficiently low $T$ the term $A_{N}$ dominates over $A_{S}$ provided the volume ratio $V_{N} / V_{S}$ is not too small. Assuming that the contribution $A_{S}$ can be neglected, from the condition ${ }^{25} A\left(T^{*}\right) \sim 1$ we arrive at the estimate for the crossover temperature $T^{*}$. above which the difference between thermodynamic potentials for even and odd ensembles is negligible and the parity effect is smeared out by thermal fluctuations. Within the logarithmic accuracy we find

$$
T^{*} \approx \varepsilon_{g}(\varphi) / \ln \left(N_{0} V_{N} \varepsilon_{g}(\varphi)\right) .
$$

In the whole temperature range $T<T^{*}$ where the parity effect remains pronounced the temperature effect on the Josephson current across SNS junction turns out to be negligible for all values of the mean free paths $\ell$. This is guaranteed by the inequality $T^{*} \ll \min \left(\varepsilon_{\mathrm{Th}}, v_{F} / d\right)$ which is always satisfied for generic systems. Hence, in order to study PC in the presence of the parity effect it is sufficient to restrict our calculation to the zero temperature limit $T=0$. In this case from the above analysis one immediately arrives at Eq. (1) which establishes a direct relation between $\mathrm{PC}$ values in the even and the odd superconducting ensembles.

Let us first evaluate $\mathrm{PC}$ for the even ensemble $I_{e}$. At $T=0$ this current identically coincides $\frac{19.20}{2}$ with one calculated for the grand canonical ensemble. The latter is easily evaluated with the aid of the quasiclassical Eilenberger equations (26). The results for the current-phase relation $I_{e}(\varphi)$ are displayed in Fig. [6] for various impurity concentrations. The dependence of the critical current $I_{C}$ on the electron mean free path $\ell$ is presented in Fig. 4

$\mathrm{PC}$ in the odd ensemble $I_{o}$ at $T=0$ can now be trivially evaluated by making use of Eq. (11) and combining our results for $I_{e}(\varphi)$ with those for the minigap $\varepsilon_{g}(\varphi)$

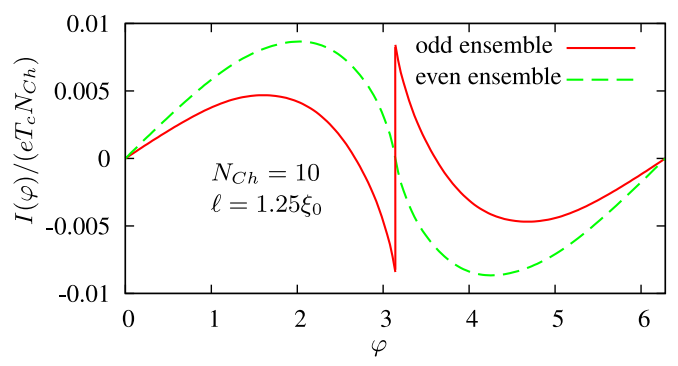

FIG. 7: Phase dependence of the Josephson current at $T=0$ for the odd and even number of electrons in the ring.

derived in the previous section. The typical dependence $I_{o}(\varphi)$ is displayed in Fig. 7 We observe that at sufficiently large values of $\varphi<\pi$ the absolute value of the odd electron contribution to PC $2 e \partial \varepsilon_{g} / \partial \varphi$ exceeds the term $I_{e}(\varphi)$ and the total current $I_{o}$ changes the sign. This non-trivial parity-affected current-phase relation is specific for SNS rings with disorder and it substantially differs from the current-phase relations derived earlier for SNS rings with ballistic $\frac{19}{19}$ and resonant ${ }^{20}$ transmissions.

At the same time, as in the previous cases ${ }^{19.20}$, in the odd ensemble there emerges a possibility for a $\pi$-junction state as well as for spontaneous currents in the ground state of the system without any externally applied magnetic flux. In order to analyze the situation it is sufficient to evaluate the ground state energy of the SNS junction by integrating Eq. (II) with respect to the phase $\varphi$. One finds

$E_{o}(\varphi)=E_{e}(\varphi)-\varepsilon_{g}(0)+\varepsilon_{g}(\varphi), \quad E_{e}(\varphi)=\frac{1}{2 e} \int_{0}^{\varphi} I_{e}(\varphi) d \varphi$,

where $E_{e / o}(\varphi)$ are the ground state energies of SNS junction for even and odd number of electrons in the ring. While the energy $E_{e}(\varphi)$ is always non-negative and reaches its minimum at $\varphi=0$, in the odd case the ground state energy $E_{o}(\varphi)$ can become negative reaching its absolute minimum at $\varphi=\pi$. This physical situation of a $\pi$-junction is illustrated in Fig. 8

It is easy to find out under which conditions the $\pi$ junction state becomes possible. For that purpose it is sufficient to observe that for any impurity concentration $E_{e}(\pi)=\alpha I_{C} / e$, where $I_{C}$ is the grand canonical critical current at $T=0$ and $\alpha$ is a number of order one which depends on the particular form of the current-phase relation. The $\pi$-junction condition $E_{o}(\pi)<0$ is equivalent to the inequality

$$
\varepsilon_{g}(0)>\alpha I_{C} / e .
$$

From Fig. [4 it is obvious that in the many channel limit the inequality (22) cannot be satisfied for sufficiently large $\ell$, in which case $I_{C}$ is large and, on the contrary, the minigap $\varepsilon_{g}(0)$ is small27. On the other hand, for sufficiently short values of the mean free path $I_{C} \propto \ell^{2}$ decays faster with decreasing $\ell$ as compared 


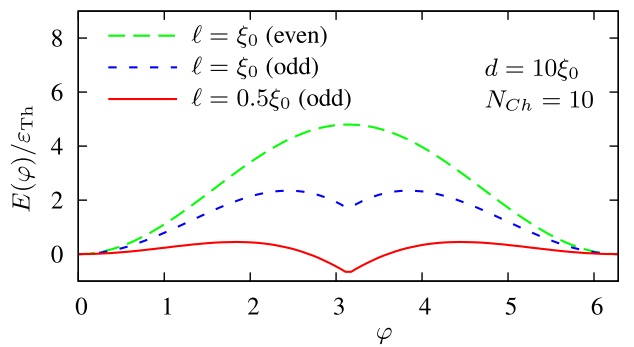

FIG. 8: Josephson energy $E(\varphi)$ of an SNS ring as a function of the phase difference $\varphi$ for the even and odd ensembles. The solid curve corresponds to a $\pi$-junction state.

to the minigap $\varepsilon_{g}(0) \propto \ell$, and the $\pi$-junction state becomes possible. In particular, in the diffusive limit one finds ${ }^{10} I_{C} \simeq 10.82 \varepsilon_{\mathrm{Th}} / e R_{N}=1.53 e N_{C h} v_{F} \ell^{2} / d^{3}$ and $\alpha \simeq 1.05$, where $R_{N}$ is the Drude resistance of a normal metal. Combining these results with the expression for the minigap ${ }^{8.13} \varepsilon_{g}(0) \simeq 3.12 \varepsilon_{\mathrm{Th}}$, from the condition (22) we observe that in the odd case a $\pi$-junction state is realized provided the number of conducting channels in the junction $N_{C h}$ is smaller than

$$
N_{C h}<0.65 d / \ell
$$

This condition is not very restrictive and it can certainly be achieved in various experiments. For sufficiently dirty junctions it allows for a formation of a $\pi$-junction state even in the many channel limit. The condition (23) can also be rewritten as

$$
g_{N}<1.73
$$

where $g_{N}=R_{q} / R_{N}=8 N_{C h} l / 3 d$ is the dimensionless conductance of a normal layer and $R_{q}=h / e^{2} \approx 25.8 \mathrm{k} \Omega$ is the quantum resistance unit.

The condition for the presence of spontaneous currents in the ground state of SNS rings with an odd number of electrons is established analogously, one should only take into account an additional energy of the magnetic field produced by $\mathrm{PC}$ circulating inside the ring. The ground state with spontaneous currents is possible provided the total energy of the ring $E_{\text {tot }}(\pi)$ becomes negative, i.e.

$$
E_{\text {tot }}(\pi)=1.8 \varepsilon_{\mathrm{Th}}\left[g_{N}-1.73\right]+\frac{\left(\Phi_{0} / 2\right)^{2}}{2 \mathcal{L}}<0,
$$

where $\mathcal{L}$ is the ring inductance. This condition is more stringent than that for the $\pi$-junction state, but can also be satisfied provided the ring inductance $\mathcal{L}$ exceeds a certain threshold value which, strictly speaking, depends on $g_{N}$ and can roughly be estimated as $\sim 0.1 \Phi_{0}^{2} / \varepsilon_{\mathrm{Th}}$.

\section{DISCUSSION}

For clarity, let us briefly summarize our key observations. Our analysis was focused on the two main issues, the proximity-induced minigap in NS and SNS structures at an arbitrary concentration of non-magnetic impurities and the effect of parity number on persistent currents in SNS nanorings. We have demonstrated that already weak disorder qualitatively modifies the density of states in the normal metal of NS and SNS proximity structures. A striking observation at this point is that the proximityinduced minigap $\varepsilon_{g}$ turns out to be isotropic for an arbitrarily weak disorder ${ }^{28}$, even though DOS at energies above the gap $\varepsilon>\varepsilon_{g}$ may remain highly anisotropic, cf., e.g., Fig. 3. Another interesting observation is the possibility of a non-monotonous dependence of the minigap $\varepsilon_{g}$ on the applied magnetic flux $\Phi$ (or phase $\varphi$ ) in SNS rings, cf. Fig. [5 Such a non-monotonous dependence can be realized only in the limit of weak disorder, whereas for not too long elastic mean free paths a monotonous decrease of the minigap from its maximum value at $\Phi=0$ to zero at $\Phi=\Phi_{0} / 2$ was found.

Piercing an SNS ring by an external magnetic flux one induces circulating persistent currents in such a ring. In an isolated ring both the amplitude and flux dependence of such PC may strongly depend on the electron parity number. Provided the number of electrons in the ring is odd, one electron remains unpaired down to $T=0$ and occupies the lowest available state above the proximityinduced minigap $\varepsilon_{g}(\varphi)$. This electron produces a countercurrent which - in the limit of relatively short electron mean free paths - may significantly modify the currentphase relation and yield a $\pi$-junction behavior and spontaneous currents in the ground state of an SNS ring flowing in the absence of any external magnetic flux. Note, that although these observations look qualitatively similar to earlier results derived for SNS rings with ballistic 19 and resonant ${ }^{20}$ transmission, there also exist important differences. In particular, the current-phase relation is entirely different in the diffusive limit considered here. Also the restriction on the number of conducting channels $N_{C h}$ in the normal metal (23) is less stringent that that formulated in Refs. 19 20. This feature of diffusive SNS rings is rather advantageous for possible experimental observation of the effects discussed here.

Our results demonstrate that superconducting parity effect can be used in order to directly measure both the magnitude and the flux dependence of the minigap in SNS nanorings. This can be done, e.g., with the aid of a setup similar to one used in Ref. 18. Such type of measurements is not restricted by the number of channels in the normal part of the ring and may serve as an alternative to tunneling spectroscopy of the minigap. Superconducting rings with embedded carbon nanotubes might be promising candidates in order to experimentally investigate parity-affected persistent currents in SNS nanorings. Several groups 29.30 .31 .32 have recently reported observations of dc Josephson current in superconducting junctions with carbon nanotubes. Therefore, it appears feasible to fabricate and experimentally investigate SNS rings with carbon nanotubes which should exhibit properties predicted and analyzed in our paper. 


\section{Acknowledgments}

We are grateful to D.S. Golubev, A.A. Golubov, M.Yu. Kupriyanov and S.V. Sharov for useful discussions. This work is supported by the European Community's Framework Programme NMP4-CT-2003-505457 ULTRA-1D "Experimental and theoretical investigation of electron transport in ultra-narrow 1-dimensional nanostructures".
1 See, e.g., P.G. de Gennes, Superconductivity of Metals and Alloys (Benjamin, New York, 1966).

2 I.O. Kulik, Zh. Eksp. Theor. Fiz. 57, 1745 (1969) [Sov. Phys. JETP 30, 944 (1970)]; C. Ishii, Progr. Theor. Phys. 44, 1525 (1970).

3 K.K. Likharev, Sov. Tech. Phys. Lett. 2, 12 (1976); A.D. Zaikin and G.F. Zharkov, Fiz. Nizk. Temp. 7, 375 (1981) [Sov. J. Low Temp. Phys. 7, 181 (1981)]; F.K. Wilhelm, A.D. Zaikin and G. Schön, J. Low Temp. Phys. 106, 305 (1997).

4 P. Dubos, H. Courtois, B. Pannetier, F.K. Wilhelm, A.D. Zaikin, and G. Schön, Phys. Rev. B 63, 064502 (2001).

5 A.D. Zaikin. Solid State Commun. 41, 533 (1982).

6 W. Belzig, C. Bruder, and G. Schön, Phys. Rev. B 53, 5727 (1996).

7 A.A. Golubov and M.Yu. Kupriyanov, J. Low Temp. Phys. 70, 83 (1988).

8 W. Belzig, C. Bruder, and G. Schön, Phys. Rev. B 54, 9443 (1996).

9 K.M. Frahm, P.W. Brouwer, J.A. Melsen, and C.W.J. Beenakker, Phys. Rev. Lett. 76, 2981 (1996).

10 W. Belzig, F.K. Wilhelm, C. Bruder, G. Schön and A.D. Zaikin, Superlatt. Microstruct. 25, 1251 (1999).

11 A.F. Andreev, Zh. Eksp. Theor. Fiz. 49, 655 (1965) [Sov. Phys. JETP 22, 455 (1966)].

12 S. Pilgram, W. Belzig, and C. Bruder, Phys. Rev. B 62, 12462 (2000).

13 F. Zhou, P. Charlat, B. Spivak, and B. Pannetier, J. Low Temp. Phys. 110, 841 (1998).

14 S. Gueron, H. Pothier, N.O. Birge, D. Esteve, and M.H. Devoret, Phys. Rev. Lett. 77, 3025 (1996).

15 D.V. Averin and Yu.V. Nazarov, Phys. Rev. Lett. 69, 1993 (1992).

16 M.T. Tuominen, J.M. Hergenrother, T.S. Tighe, and M. Tinkham, Phys. Rev. Lett. 69, 1997 (1992).

17 G. Schön and A.D. Zaikin, Europhys. Lett., 26, 695 (1994).

18 P. Lafarge, P. Joyez, D. Esteve, C. Urbina, and M.H. Devoret, Phys. Rev. Lett. 70, 994 (1993).

19 S.V. Sharov and A.D. Zaikin, Phys. Rev. B 71, 014518
(2005).

20 S.V. Sharov and A.D. Zaikin, Physica E 29, 360 (2005).

21 G. Eilenberger, Z. Phys. 214, 195 (1968).

22 Generalization of the analysis to nanostructures with few conducting channels and impurity scattering requires, in general, going beyond the quasiclassical Eilenberger formalism, see A.V. Galaktionov and A.D. Zaikin, Phys. Rev. B 65, 184507 (2002).

23 The maximum value of the electron mean free path used in our numerical calculation was $l=80 \xi_{0}$.

24 B. Janko, A. Smith, and V. Ambegaokar, Phys. Rev. B 50, 1152 (1994).

25 D.S. Golubev and A.D. Zaikin, Phys. Lett. A 195, 380 (1994).

26 D.V. Averin and Yu.V. Nazarov, Physica B 203, 310 (1994).

27 It is worth stressing again that in the quasi-ballistic the minigap is small only in the many channel limit, while for SNS juctions with few conducting channels the electron motion in the transversal direction is quantized and the minigap remains non-zero also in the ballistic limit. In this respect the situation considered here differs drastically from that addressed in Refs. 1920.

28 Effective isotropization of some other quantities in hybrid proximity structures can also be expected already in the weak disorder limit. For instance, an angleindependent decay length of the Cooper pair amplitude in SFS (superconductor-ferromagnet-superconductor) structures with disorder has been found, see D.Yu. Gusakova, M.Yu. Kupriyanov, and A.A. Golubov, cond-mat/0605137

29 P. Jarillo-Herrero, J.A. van Dam, and L.P. Kouwenhoven, Nature, 439, 953 (2006).

30 H.I. Jorgensen, K. Grove-Rasmussen, T. Novotny, K. Flensberg, and P.E. Lindelof, cond-mat/0510200

31 K. Grove-Rasmussen, H.I. Jorgensen, and P.E. Lindelof, cond-mat/0601371

32 P. Hakonen, private communication. 